\title{
EL FEMINISMO CONSTITUCIONALISTA EN CONSTRUCCIÓN
}

\section{FEMINIST CONSTITUTIONALISM UNDER CONSTRUCTION}

Olga Patricia Velásquez Ocampo(1)

Universidad de los Andes, Colombia

Resumen: Este artículo propone un esquema del feminismo constitucionalista, brindando un marco a partir de los desarrollos en diseño e interpretación constitucional y de lo que se puede crear a partir de dicha teoría. Los argumentos que proceden de un sector de la academia a favor de la inclusión del enfoque de género ofrecen una nueva luz para la solución de problemas actuales, dentro del marco del derecho constitucional. Estas maneras de intervenir en el derecho constitucional buscan transformaciones sociales y políticas para las mujeres, a través de herramientas como el diseño constitucional, las reformas constitucionales, la interpretación constitucional, entre otras. Todas estas intervenciones desafían las posiciones tradicionales del derecho. De esta forma las antiguas y nuevas constituciones cambian sus formas para adaptarse a un mundo dinámico que demanda un trato diferente para las mujeres.

Palabras clave: Feminismo constitucionalista, constitución, diseño constitucional, jurisprudencia constitucional, interpretación constitucional, inclusión.

Abstract: This article proposes a scheme of constitutional feminism, providing a framework based on the developments in constitutional design and constitutional interpretation and the possibilities that could be created from that theory. The arguments that are exposed by part of the academy in favor of the inclusion of the gender approach offer a new light for the solution of existing problems within the framework of constitutional law. These ways of intervening in constitutional law seek social and political transformations for women, using tools such as the constitutional design, constitutional amendments, constitutional interpretation, among others. All these interventions challenge the traditional positions of the

(1) Candidata a Doctora en Derecho de la Universidad de Los Andes, Bogotá, Colombia. Abogada y Especialista en Derecho Público de la Universdiad EAFIT, Medellín. Magister en Derecho de la Universidad de Los Andes. Email: op.velasquez10@uniandes.edu.co 
law, and that is how the old and new constitutions change in order to adapt in a dynamic world, that demands different treatment for the women.

Key words: Constitutional feminism, constitution, constitutional design, constitutional jurisprudence, constitutional interpretation, inclusion. 


\section{Introducción}

Las constituciones representan compromisos acordados por personas con ideas y necesidades diferentes, las cuales permiten que todas ellas puedan coexistir $^{(2)}$. Pero la inclusión del enfoque de género en el constitucionalismo hace que surjan nuevas y urgentes cuestiones, a saber: ¿Qué pasa cuando una gran parte de la población que es gobernada por la Constitución no participó de su creación? ¿Cómo es posible la inclusión de las mujeres y a través de qué medios? Cuando se entiende que una de las virtudes de una Carta Constitucional es su dificultad para ser modificada, ¿cómo incluir a las mujeres dentro de un pacto en el que no participaron? ¿Qué ocurre cuando las mujeres fueron incluidas en el pacto, habiendo entonces participado de él, pero sus reglas no les son efectivamente aplicadas? Estos son interrogantes que requieren respuestas diferentes a las que se ha dado desde el constitucionalismo tradicional y deben ser por lo tanto abordadas desde un espacio de discusión incluyente, como el feminismo constitucionalista.

En este trabajo busco dar cuenta de las intervenciones del feminismo al derecho constitucional. Para esto estudiaré qué buscan las feministas con el uso del derecho constitucional $y$, abordaré preguntas como: ¿Es posible identificar qué cambios quieren? ¿Por qué es beneficiosa la intervención de las mujeres en los procesos constitucionales? ¿Cuándo es beneficiosa esa intervención? ¿Qué estrategias constitucionales pueden implementarse de acuerdo con el contexto social? y ¿cuáles son los resultados que se requieren para cambiar la situación de discriminación o dominación estructural de las mujeres?

Para responder a estos interrogantes, presento el artículo de la siguiente manera. En primer lugar, haré referencia al concepto de feminismo constitucionalista y sus diferentes usos; luego expondré las rutas de las intervenciones feministas al presentar nuevos enfoques en antiguos reclamos sobre derechos de las mujeres.

Sostendré que es posible detectar dos formas de comunicación entre las feministas que usan el derecho como herramienta para alentar el cambio social y el derecho constitucional. Un camino es a través del diseño constitucional, es decir, de la participación de las mujeres en la creación de nuevas constituciones. Este

(2) *Este artículo es el resultado del proceso de investigación realizado para el proyecto de Monografía de Grado de la Maestría en Derecho de la Universidad de los Andes, Bogotá.

**Magister en Derecho de la Universidad de los Andes, Bogotá. Estudiante del Doctorado en Derecho, Universidad de los Andes, Bogotá. op.velasquez10@uniandes.edu.co

"Constitutions can and do embody compromises that are necessary for large groups of people with different ideas to live together". En: Jackson, V. C. (January 01, 2009). Gender Equality and the Idea of a Constitution: Entrenchment, Jurisdiction, Interpretation: Conclusion. Constituting Equality: Gender Equality and Comparative Constitutional Rights, P. 321. 
proceso incluye la propuesta del texto constitucional, la discusión sobre los temas que serán incluidos y la ejecución de lo plasmado en la carta constitucional. Un segundo camino es el de la interpretación constitucional donde se hace un ejercicio propositivo por parte de las feministas que encuentran una manera de alcanzar los objetivos de igualdad y no discriminación a través de la decisión judicial, en otras palabras, proponen argumentos que permiten que los y las jueces acojan formas diferentes de estudiar la situación de las mujeres.

Las preguntas que se presentan en este trabajo buscan promover una lectura de la constitución que tenga de presente el desafío de inclusión e igualdad que demandan las mujeres, y mostrar dos caminos (de muchos posibles) para lograr avances en la agenda de las mujeres. ${ }^{(3)}$

\section{Feminismo constitucionalista}

De manera reciente, algunos proyectos académicos han propuesto el término feminismo constitucionalista como una forma de explorar la relación entre el derecho constitucional y el feminismo. Lo anterior se ha hecho a través de evaluaciones, desafíos y de la redefinición de la idea misma del constitucionalismo desde una perspectiva feminista ${ }^{(4)}$. Otra versión del constitucionalismo feminista lo define como "el empleo de los poderes y disposiciones constitucionales para mejorar las condiciones de las mujeres" (Ishwara Bhatt, 2001). En años recientes, se ha entendido el concepto de feminismo constitucionalista como "parte del constitucionalismo crítico" teniendo en consideración "los graves conflictos sociales y se compromete a comprenderlos con una finalidad "liberalizadora y emancipadora". En palabras de Garay, este constitucionalismo "estudia cómo el patriarcado ha coexistido y coexiste con todos los sistemas económicos (esclavismo, feudalismo y capitalismo) determinando la distribución del poder y las contradicciones de la igualdad formal." Garay parte de la igualdad como eje central del estado constitucional. En el espacio del derecho constitucional, estas académicas consideran que el constitucionalismo con enfoque de género propone un proyecto de cambio, que busca, “... superar las relaciones de género y alcanzar la convivencia pacífica sobre la base de relaciones realmente igualitarias de mujeres y hombres."(5)

(3) Sobre posibles caminos alternativos que ofrece el feminismo constitucionalista, ver: Beverley Baines, Ruth Rubio-Marin, Peter Oliver, Patrick Macklem, \& Nathalie Des Rosiers. (2017). Part VI Constitutional Theory, C Key Debates in Constitutional Theory, Ch.45 Feminist Constitutionalism in Canada. In The Oxford Handbook of the Canadian Constitution (Oxford Handbooks, p. Oxford Handbooks, The Oxford Handbook of the Canadian Constitution). Oxford University Press.

(4) What is feminist constitutionalism? Basically, it is the project of rethinking constitutional law in a manner that addresses and reflects feminist thought and experience. En: Beverley Baines, Daphne Barak-Erez y Tsvi Kahana, op. cit. Pág. 1.

(5) N. Garay (2013). Las Declaraciones de los Derechos de la Mujer y la Ciudadana de 1791 y de Independencia de Haití de 1804 en el proceso de positivización de los derechos fundamentales. Aportes desde el constitucionalismo feminista, en Revista Letras Jurídicas, 28, México: Universidad Veracruzana. 
En este sentido, desde diferentes partes del globo han surgido voces que abogan por cambios. Diferentes corrientes feministas han hecho esfuerzos por la promoción de una suerte de auditoria de género al proceso constitucional, que sea atento a las preguntas constitutivas de este, los mecanismos de participación y el lugar que ocupan las mujeres dentro del sistema de gobierno, con un objetivo específico: ir más allá de los derechos, a través del énfasis en las estructuras constitucionales (Irving, 2008). De la misma manera las feministas han abordado problemas que afectan a las mujeres usando el litigio como estrategia para lograr cambios a través de las Cortes ${ }^{(6)}$.

Es esta preocupación la que conduce a las mujeres a pensar en caminos hacia la inclusión. El diseño constitucional se presenta entonces como una vía de intervención a través de la participación de las mujeres en los procesos constitucionales. Pero para que esta intervención sea efectiva, se requiere de un verdadero compromiso con los avances para mejorar la situación de las mujeres, y en muchos casos, del interés y disposición de estas para involucrarse con actividades de carácter político.

En este contexto, la necesidad de crear espacios de participación para las mujeres se plantea como una meta de los actuales procesos de reforma constitucional. La inclusión de las mujeres ${ }^{(7)}$ en este tipo de procesos permite crear una sociedad más justa y democrática, especialmente en aquellos lugares donde ellas se han visto afectadas por los rigores de la guerra, o en casos en que su antigua constitución no tenía en cuenta sus derechos, ni mucho menos su inclusión en términos de igualdad. Curiosamente, a pesar de que las mujeres constituyen mayoría en la sociedad, existe una tendencia a ignorarlas en los procesos constitucionales $\mathrm{o}$, de manera frecuente, a que estas sean reacias a participar en la construcción constitucional.

Algunos ejemplos de lo anterior incluyen el caso de Siria ${ }^{(8)}$, donde se reportó que las mujeres rechazaron oportunidades de liderazgo político debido a su falsa creencia de que hombres estarían mejor preparados para estas funciones. Así mismo, en Liberia, las mujeres que desempeñaron papeles esenciales durante la guerra, al terminar el conflicto se apartaron a sus roles tradicionales en el hogar, interesándose

((6)) Para información acerca de diferentes casos abordados por diferentes clases de feministas que emplean el litigio como estrategia para avanzar en la agenda de las mujeres ver: Barak-Erez, D. (2012). Her-meneutics. Feminist constitutionalism: Global perspectives, 85-97.

(7) En este sentido, el informe de IDEA sobre los Grupos Marginados y la Construcción Constitucional muestra el rol de las mujeres que logran superar los obstáculos para llegar a una posición dentro del proceso de diseño constitucional es vital para promover mejores condiciones en situaciones posteriores a la construcción constitucional.

(8) Sobre los casos de Siria y Liberia, ver: International IDEA, Marginalized Groups and Constitution Building (Stockholm: International IDEA, 2014). 
solo en cuestiones de supervivencia. Además, en el diseño de la Constitución de Japón de 1946, mujeres como Beate Sirota, participaron del proceso constitucional sin contar con la formación adecuada, ella fue comisionada para diseñar las garantías constitucionales relativas a las mujeres, mientras que otras provisiones constitucionales fueron proyectadas por expertos en la materia. De acuerdo con Laura Lucas, este es un rasgo del diseño constitucional: la presunción de que las mujeres deben ser protegidas pero que los temas que realmente las involucran no son lo suficientemente complejos como para demandar la presencia de especialistas que diseñen el marco constitucional para ellas (Lucas, 2009:133 a 166).

Estos proyectos son difundidos para la creación de una agenda constitucional feminista (Baines y Rubio- Marín, 2012). Una agenda que propone estrategias que incluyan las preocupaciones de las mujeres afrodescendientes, pobres, y otros sectores marginados ${ }^{(\mathbf{9})}$.

En este punto es importante resaltar que históricamente los procesos y textos constitucionales han fallado en reflejar las preocupaciones de las mujeres y otros grupos excluidos. Por esto la historia de las constituciones puede verse como una de la categorización y exclusión de ciertos sectores de la población, ya sea a través de proyectos imperiales o de manera interna a través de la construcción de la ciudadanía. El impacto diferenciado que las constituciones tienen en las vidas de los hombres y las mujeres es una característica que ha sido suficientemente estudiada y reportada, mostrando que ${ }^{(10)}$ la propia naturaleza del constitucionalismo está en entre dicho y levantando dudas sobre si como las constituciones pueden servir a las mujeres como colectividad.

\section{La ruta hacia la inclusión}

Como he sugerido en las líneas anteriores, los aportes feministas han tenido gran impacto en el constitucionalismo y en otras ramas del derecho. Sin embargo, en el campo de la teoría de la interpretación constitucional, por lo menos en Estados Unidos, los diálogos entre la teoría legal feminista y el derecho constitucional no han sido fluidos. Los trabajos sobre teoría constitucional e interpretación se enfocan en aspectos "positivistas y normativos", explicando para qué sirven las constituciones, y como deben ser interpretadas.

Así lo señala Vicki C. Jackson ${ }^{(11)}$ al citar el trabajo de Richard H. Fallon, Jr.,

(9) Dependiendo de la corriente feminista de la que se sea partidaria, dependerán las intenciones de las intervenciones. Ver: Barak-Erez, D. (2012). Her-meneutics, op. cit., pp. 86-90.

(10) Sobre el impacto diferenciado de las constituciones en el mundo, ver: Laura Lucas y Taryn Marks, The Women and Constitutions Project: http://law.duke.edu/capstone/constitutionaldesign.html

(11) Vicki C. Jackson cita la obra de Richard H. Fallon, Jr. : How to Choose a Constitutional Theory, 87 Calif. L. Rev. 535 (1999). Op. cit. Pág. 315. 
Jackson sostiene que Fallon incluye una gran cantidad de autores referentes en la teoría constitucional, como Richard Posner, Bruce Ackerman, Ronald Dworkin, Laurence Tribe, entre otros, pero que ninguna feminista (académica o no) es mencionada. Esta ausencia no es justificada, ya que existen investigaciones como las de Robin West, Catharine Mackinnon, Vicky Jackson, Helen Irving, Alexandra Dobrowolsky, Vivien Hart, Tracy Higgins, Beverley Baines, Daphne Barak-Erez (12), entre otras feministas, que han explorado el campo del derecho constitucional con una mirada incluyente, proponiendo diferentes estrategias para avanzar en la agenda feminista a través del derecho constitucional. Ciertamente las intervenciones feministas en el derecho constitucional se han manifiestado de múltiples formas, por ejemplo a través de la discusión de temas específicos como el aborto, las acciones afirmativas o el acoso sexual. Sin embargo, es necesario que el feminismo constitucionalista sea empleado como un enfoque para analizar plenamente el texto constitucional.

Como mencioné arriba, al analizar las intervenciones feministas es posible trazar dos caminos. El primero es el del diseño constitucional, como una de las intervenciones del feminismo en la construcción y ejecución de las cartas constitucionales. El segundo, es el uso del espacio judicial, como campo de inauguración, desarrollo y ejecución de la agenda feminista. A continuación presentaré las características principales de estas dos vías.

\subsection{El diseño constitucional y las mujeres}

Después de la Segunda Guerra Mundial, numerosos países emprendieron el camino hacia la reconstrucción de sus acuerdos constitucionales ${ }^{(13)}$. El

(12) Ver: Robin L. West, Constitutional Scepticism, 72 B.U. L. REv. 765, 780-92 (1992); Robin West, Progressive and Conservative Constitutionalism; Catharine Mackinnon, Foreword, (2012). Feminist constitutionalism: Global perspectives. Cambridge: Cambridge University Press; Catharine Mackinnon, Michel, R., en András, S., \& Catharine, A. M. (May 17, 2012). Gender in Constitutions; Vicky Jackson, 'Conclusion: Gender Equality and the Idea of a Constitution: Entrenchment, Jurisdiction, Interpretation', en Susan H. Williams (ed.), Constituting Equality: Gender Equality and Comparative Constitutional Law, Cambridge University Press; Helen Irving, (2008). Gender and the constitution: Equity and agency in comparative constitutional design. Cambridge: Cambridge University Press; Alexandra Dobrowolsky \& Vivien Hart, Women making constitutions: New politics and comparative perspectives. Houndsmill, Basingstoke, Hampshire: Palgrave Macmillan; Tracy Higgins, (January 01, 1997). Democracy and Feminism. Harvard Law Review, 110, 8, 1657; Beverley Baines \& Daphne Barak-Erez, (2012). Feminist constitutionalism: Global perspectives, 85-97. (13) La igualdad de género y los derechos de las mujeres fueron reconocidos en numerosos textos constitucionales en el periodo entre guerras. Sin embargo, es poco probable que las mujeres hayan participado de manera activa en los procesos constitucionales antes de la Segunda Guerra Mundial. Algunos autores resaltan la existencia de una participación activa de las mujeres en el diseño constitucional, si bien no de manera colectiva (movimientos) existen registros de su participación a través de observaciones individuales, y discusiones entre grupos de mujeres durante la época de construcción constitucional norteamericana, a través de aplicaciones o peticiones de las mujeres a las convenciones constitucionales o a las comisiones de redacción, de campañas para enmiendas constitucionales, entre otras. Akhil Reed Amar, "Women and the Constitution" (1994-1995) 18 Harvard Journal of Law and Public Policy 465. 
incremento de la actividad constitucional generó un debate sobre gobernanza, los derechos humanos y el reconocimiento de la diversidad en la sociedad. Debido a que muchos de estos procesos deliberativos aún se mantienen en movimiento y teniendo en cuenta que las discusiones sobre la naturaleza y formas de la democracia continúan, la política constitucional se ha convertido en parte fundamental (y lo seguirá siendo) de la esfera política en el siglo XXI (Dobrowolsky y Hart, 2003:1). Esto es particularmente cierto para aquellos Estados que vieron en la construcción constitucional un paso necesario en la transición hacia la democracia.

Con más de cien revisiones constitucionales y numerosas nuevas constituciones adoptadas en los últimos veinte años (Hart, 2003), se puede afirmar que el derecho constitucional es unárea en crecimiento.Los artículos constitucionales que versan sobre la igualdad de género se difunden ampliamente ${ }^{(14)}$ en conjunto con los mecanismos para promoverla. Asimismo, hay una protección del estatus de los derechos de las mujeres a través de instrumentos legales internacionales ${ }^{(15)}$.

Este auge en el desarrollo de las constituciones demanda la participación de las mujeres en las discusiones sobre los derechos de los que serán titulares, la forma en la que los mismos serán garantizados y, ciertamente, la inclusión de la mujer en el espacio del diseño constitucional, espacio en el que ha permanecido al margen y en el que, la inequidad y la subordinación de las mujeres han sido rasgos característicos.

Entre las académicas que estudian el diseño constitucional ${ }^{(16)}$ desde un enfoque que privilegia el lugar de lo femenino en las discusiones constitucionales y las ubica en un lugar central en el diseño, se encuentran autoras como Helen Irving, Vivien Hart, Alexandra Dobrowolsky y Paula Monopoli ${ }^{\left({ }^{17)}\right.}$. Estas autoras consideran el diseño constitucional como un espacio donde se plantean discusiones relacionadas con la vida y el derecho, con lo qué es y lo qué quiere la sociedad (Morgan y Alzate Buitrago, 1992: 370 a 371). Y es que a medida que los grupos tradicionalmente excluidos, como las mujeres, comienzan a participar en la creación de nuevas

(14) Los efectos de estos diseños constitucionales y las nuevas reformas internacionales se encuentran lejos de la homogeneidad y proveen caminos para futuras investigaciones. Ver: Dobrowolsky y Hart (2003). (15) Algunos ejemplos de estos instrumentos son la CEDAW (1979); que establece la violación como un crimen de guerra en el derecho internacional; la decisión Akayesu del Tribunal Penal Internacional para Ruanda en 1998; la Resolución 1820 de 2008 de las Naciones Unidas que vincula explícitamente la violencia sexual como táctica de guerra con la mujer, la paz y la seguridad.

(16) El diseño constitucional es apenas una de las estrategias que existe para la inclusión de las mujeres. Ver:Waylen, G. (January 01, 2006). Constitutional engineering: What opportunities for the enhancement of gender rights? Third World Quarterly, 277, 1209-1221.

(17) En este grupo podemos ubicar las obras de Helen Irving, principalmente en Where have all the women gone? Gender and the literature on constitutional design, Drafting, design and gender. La sección de la academia dedicada al feminismo está compuesta de manera casi exclusiva por mujeres, Tsvi Kahana (coeditor del libro Feminist Constitutionalism Global Perspectives es el único hombre que contribuye en el citado texto). En este artículo solo otro hombre hace una contribución al tema. 
constituciones, factores que no habían sido incluidos deben empezar a ser tenidos en cuenta. Temas antes juzgados como tabú entran a ser parte importante en las discusiones, cambiando de manera sustantiva el texto constitucional ${ }^{(\mathbf{1 8})}$.

Las ideas sobre la necesidad de cambios constitucionales para incluir las voces femeninas tienen antecedentes más o menos remotos. Ejemplos de ello son la Vindicación de los derechos de la mujer (1792) de Mary Wollstonecraft ${ }^{(19)}$ y la Declaración de los Derechos de la Mujer y de la Ciudadana (1791) de Olympe de Gouges ${ }^{(20)}$. También, el caso de Abigail Adams ${ }^{(21)}$, esposa de John Adams, de quien se dice que trató de influenciar a su marido con ideas sobre el rol de la mujer en una sociedad post revolucionaria y le pidió que recordara a las mujeres en la Constitución Norteamericana. Lo que podemos encontrar hoy es que a pesar de sus insistencias en aquel momento, las voces femeninas fueron olvidadas. Sin embargo, la movilización de las mujeres en este periodo demuestra su interés por la arquitectura constitucional, así como, su esperanza en la capacidad de los textos constitucionales para transformar la realidad, ánimos que luego habrían de renacer con la reconstrucción constitucional.

De esta forma, se puede decir que la preocupación de las mujeres por intervenir en los debates constitucionales en los Estados Unidos se remonta a la segunda mitad del siglo XVIII (Irving, 2011: 92). En esta época las norteamericanas presentaron (sin mayores resultados) observaciones sobre la extensión de la protección de la Enmienda XIV -cláusula de igual protección entre todas las personas - en temas relacionados con la práctica de la profesión y el voto para las

(18) Como lo afirma Owen Fiss, el feminismo tiene distintos significados para distintas personas, existiendo feministas radicales, culturales, liberales y socialistas. Helen Irving señala que “... al existir diferentes alternativas (en algunas ocasiones irreconciliables) del feminismo hace que la tarea de alcanzar un diseño constitucional igualitario de género (y hasta la declaración de la "igualdad" como la meta) extremadamente difícil." Lo cual se presenta como una de las dificultades en el ámbito del diseño constitucional. Irving, H. (January 01, 2011). Drafting, Design and Gender. Comparative Constitutional Law, 1937; Irving, H. Volume 4(2), 2011, pág. 33.

(19) Incluso si este trabajo hubiera sido publicado algunos años antes, la probabilidad de que este influyera en los redactores de la constitución de Filadelfia o en los delegados estatales encargados de la ratificación es poca. Sin embargo, el trabajo de Wollstonecraft inspiro las campañas de las mujeres en épocas subsiguientes. Ver: Helen Irving, Gender And The Constitution, op.cit., p. 6

(20) El trabajo de esta autora es una versión de la Declaración de los Derechos del Hombre y del Ciudadano, donde incluye o adiciona la palabra mujer, en defensa de la autonomía reproductiva de las mujeres, la igualdad de hombres y mujeres, entre otros. Cfr. Helen Irving, Gender And The Constitution, op.cit., p. 7 (21) Abigail Adams defensora de los derechos de las mujeres y esposa de John Adams, segundo Presidente de los Estados Unidos (1797-1801), y miembro del Comité de los Cinco encargado de la redacción de la Declaración de Independencia del mismo país. Carta de Abigail Smith Adams a John Adams (Mar. 31, 1776), en Adams, J., Adams, A., \& Adams, C. F. (1876). Familiar letters of John Adams and his wife Abigail Adams, during the Revolution: With a memoir of Mrs. Adams. New York: Hurd and Houghton. Y Agosín, M. (2001). Women, gender, and human rights: A global perspective. New Brunswick, NJ: Rutgers University Press. Pág. 25. 
mujeres ${ }^{\text {(22) }}$. A pesar del fracaso de las primeras intervenciones, fue gracias a estas campañas que en 1920 las norteamericanas lograron el derecho al voto femenino consagrado en la Enmienda XIX.

Vale la pena aclarar que las feministas preocupadas por el diseño constitucional observan tanto los procesos que llevan a la creación o renovación de las constituciones como el producto final de las discusiones propias de estos procesos. Es decir, no dejan de lado la posibilidad de hacer exigibles las disposiciones constitucionales. Sin embargo, se enfrentan al reto de comprender ¿Cuáles son los resultados reales de las constituciones?

En este sentido, es útil pensar en el diseño constitucional desde la óptica feminista a partir de tres preguntas: (i) ¿cómo se organizan las mujeres para participar en el diseño constitucional?, (ii) ¿qué quieren incluir las mujeres en la Constitución?,

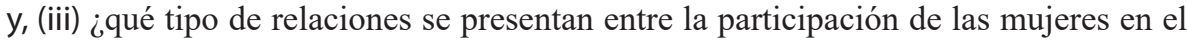
proceso constitucional, el texto producido y los cambios en las condiciones de las mujeres después de ser adoptada la nueva Constitución?

Estos interrogantes nos permiten tener una visión del panorama al que se enfrentan los constitucionalistas que buscan la incorporación activa de las mujeres en los campos político y social, a través de su intervención en la construcción constitucional. Las respuestas para las preguntas planteadas dependerán del estudio juicioso de los países que conduzcan renovaciones constitucionales, observando de cerca el diseño, proceso y materialización de las consignas propuestas por y para las mujeres.

Así, autoras como Helen Irving parten de la idea de que las constituciones no son neutrales al género (Irving, 2008). La comprensión de las constituciones como impactadas por el género y el reconocimiento de las diferencias entre hombres y mujeres presentes en los textos constitucionales ha sido el resultado del esfuerzo de una variedad de organizaciones. Por un lado, la idea del impacto del género es apoyada por una porción significativa de la literatura sobre mujeres, constitución, interpretación y cambio constitucional (Mackinnon, 2012: 397416). Por el otro, las variadas formas en las que operan las constituciones para los hombres y las mujeres pueden evidenciarse, por ejemplo, en las consideraciones acerca de quién tiene ciudadanía y quién no. Esto es importante porque las constituciones distribuyen poder -estableciendo quién lo ejerce y dónde- lo cual produce un impacto diferencial para las mujeres. Además, las constituciones,

(22) Para una breve descripción acerca de la historia de las mujeres y la Constitución de Estados Unidos, ver: Akhil Reed Amar, "Women and the Constitution" (1994-1995) 18 Harvard Journal of Law and Public Policy 465, Samantha Ricci, "Rethinking Women and the Constitution: An Historical Argument for Recognizing Constitutional Flexibility with Regards to Women in the New Republic" (2009) William \& Mary Journal of Women and the Law, Vol. 16. Artículo 8, 205-235. 
establecen los detalles sobre la forma en que se habrán de conducir las relaciones estado-ciudadano, y regula las relaciones entre la ciudadanía.

El interés de las mencionadas académicas por el diseño de la constitución ha radicado en la necesidad de estudiar y cambiar la falta de participación e inclusión de las mujeres tanto en el proceso de producción constitucional como en los enunciados constitucionales mismos. Bajo este entendido, este es un fenómeno que debe alterarse para estar a la par con un mundo que se encuentra en constante cambio. Para estas feministas, el proceso a través del cual se escriben las constituciones es tan importante como el producto final (Hart, 2003).

Si bien, como señala Irving, la literatura sobre el proceso constitucional (constitution making) es sustancial, hasta hace muy poco se empezó a prestar atención al género como factor vital del diseño ${ }^{(23)}$. Por ejemplo, en Principles of Constitutional Design, Donald Lutz presenta su trabajo como:

"un libro para cualquiera en cualquier lugar sentado escribiendo una constitución. [...] diseñado para ser educativo incluso para aquellos que no se encuentran familiarizados con el diseño constitucional pero que quieren tener un mejor entendimiento de la naturaleza y problemas del constitucionalismo y sus piedras angulares--- de manera particular la soberanía y la separación de poderes."

No obstante estas promesas, el texto de Lutz no incluye mención alguna a la participación de las mujeres en el diseño constitucional ${ }^{(24)}$. Hoy por hoy, existen numerosos estudios acerca del diseño. Pero a pesar de la abundante literatura, el tema de género desaparece como referente, y las mujeres, en caso de ser reconocidas, solo se enumeran como un subconjunto más dentro de los desafíos del diseño constitucional ${ }^{(25)}$.

Pero la falta de publicaciones sobre el tema no debe considerarse como un indicador para determinar la menor importancia ${ }^{(26)}$ del diseño constitucional, pero si debe ser considerada como una señal de la necesidad de una mayor

(23) Véase Irving, H. (January 01, 2011). Drafting, Design and Gender. Comparative Constitutional Law, 19-37; Irving, H. Volume 4(2), 2011. Where have all the women gone? Gender and the literature on constitutional design. Contemporary Readings in Law and Social Justice, pp. 89-115.

(24) (Lutz, 2006, Principles of Constitutional Design, traducción propia). En igual sentido, Cass Susntein hace referencia "diseño" en el nombre de su trabajo (2001), pero la discusión de género descrita por el autor se dedica al análisis de la validez de las leyes para restringir o no la protección de la igualdad. Sunstein, Cass R. (2001), Designing Democracy: What Constitutions Do, Oxford University Press.

(25) En las investigaciones sobre identidad constitucional, legitimidad, poder constituyente, y temas relacionados con el diseño constitucional, el género es ignorado casi por completo.

(26) La doctrina actual desarrolla casi de manera exclusiva el tema del diseño constitucional y las mujeres, empleando el término "género" como sinónimo de "mujer", algunas de las obras incluidas dentro de esta investigación fueron rastreadas a partir del uso de la palabra género en reemplazo de mujer. 
intervención en este campo. En otras materias, como las investigaciones sobre los asuntos electorales, el género es más importante, por ejemplo, para hablar de sistemas de cuotas, siendo este uno de los ejemplos típicos de la intervención en la arquitectura constitucional.

Asimismo, al hablar sobre temas relacionados con la paz mundial y las mujeres, los académicos proponen de manera constante la inclusión de la perspectiva feminista en casi cualquiera de los aspectos relevantes del orden político global o constitucional. Para estos académicos, aceptar las normas patriarcales en casi todas las culturas ha permitido tanto la opresión de las mujeres como la militarización de los estados, siendo esto contrario a lo que significa un verdadero cambio que conduzca a un avance hacia la globalización, donde la dignidad humana garantice que todas las personas sean sujetos y electores de su propio destino. Esta es la norma básica que las feministas han buscado establecer para las mujeres y todos los grupos oprimidos.

La confianza que las mujeres tienen en el poder transformador de la constitución ${ }^{(27)}$ refuerza la urgencia de su participación en el diseño constitucional ${ }^{(\mathbf{2 8})}$, es por ello que las corrientes feministas han trabajado para efectuar cambios sociales y políticos a través de las reformas constitucionales ${ }^{(29)}$.

Siguiendo a Irving, cuando se habla sobre género y diseño constitucional, es preciso contemplar el alcance de las palabras que serán puestas en la constitución, siendo posible con esto, adelantarse a las diferentes interpretaciones que una Corte Constitucional pueda ofrecer (esto, teniendo en cuenta que dicha institución también debe ser plasmada en la constitución) ${ }^{(\mathbf{3 0})}$. Es por ello que la participación de las mujeres en el proceso constitucional no es el final del camino, la constitución puede ser alterada o mal interpretada en cualquier momento, si no se mantiene bajo constante vigilancia. Un ejemplo de esto es que, a pesar de la incorporación de cláusulas de igualdad en las constituciones, estas excluyen (y han excluido) los derechos de las mujeres en diferentes áreas.

La intervención de las mujeres en el derecho constitucional a través del diseño constitucional permite contar con la posibilidad de hacer una constitución

(27) Un sector de la academia percibe que "las constituciones no son panaceas que puede curar problemas sociales, económicos y políticos fundamentales..." En: Keith S. Rosenn, The Success of Constitutionalism in the United States and Its Failure in Latin America: An Explanation, 22 U. MIAMI INTER-AM. L. REV. 1, 26 (1990). Pero la apuesta de las constitucionalistas citadas es creer en el cambio que nace con la renovación.

(28) Si bien existe un sector de mujeres que no cree en el texto constitucional, cuando están en proceso de reformas o nuevas constituciones, la mayoría de las mujeres considera que lo mejor es participar.

(29) Dobrowolsky, A. Z. (2003). Women, Constitutionalism and Contestation: Some Tentative Conclusions. Women making constitutions: New politics and comparative perspectives. Houndsmill, Basingstoke, Hampshire: Palgrave Macmillan

(30) Cfr. Irving, H. (2011). Drafting, Design and Gender. Comparative Constitutional Law, 19-37. 
incluyente, donde de antemano se establezcan mecanismos que permitan asegurar la protección de las mujeres a través de provisiones concretas que no conlleven a discusiones posteriores sobre el sentido de la norma. Esta dificultad en particular, la imposibilidad de participar en un proceso constitucional desde cero, puede ser un obstáculo para las feministas que intervienen en constituciones preexistentes, quienes deben acudir a otras maneras de participación, ya sea a través de reformas o ante la rama judicial.

La necesidad de caminos alternos para asegurar la inclusión de las mujeres en el derecho constitucional es ejemplificada por Hart y Dobrowolsky ${ }^{(31)}$ al indicar que las mujeres deben repartir sus esfuerzos entre el diseño constitucional, los impulsos en el campo de la política y la legislación. Además de un camino que ha sido recorrido previo al boom de las constituciones, la vía del litigio ante las cortes.

\subsection{La interpretación judicial como camino alternativo al diseño constitucional}

Las decisiones relativas al diseño del texto constitucional traen consigo la preocupación sobre el sentido e interpretación que las provisiones constitucionales puedan tener. La discusión acerca de la interpretación constitucional se encuentra abierta debido a los múltiples sentidos que pueden darse a las disposiciones constitucionales.

Para resolver esta dificultad existen numerosas metodologías de interpretación, esta variedad puede encontrarse en la mayoría de los países donde existe el judicial review. Sin importar qué método se adopte es frecuente optar por una alternativa que favorezca una interpretación apegada al texto de la norma. En otras palabras, no existe una técnica general para la interpretación de la constitución, desde ninguna perspectiva, incluyendo la visión feminista (Irving, 2008: 27). Hasta finales de los años noventa, muy poca investigación acerca de la interpretación constitucional con enfoque en temas relacionados con los derechos de las mujeres había sido realizada ${ }^{(32)}$. En el campo del derecho constitucional las críticas feministas no tienen el impacto deseado, el foco central de los trabajos más citados en teoría constitucional e interpretación se concentran en enseñar un panorama sobre la función de las constituciones y cómo estas deben ser interpretadas.

(31) "when women put all their eggs in one basket, there is a greater chance that their constitutional efforts can become scrambled." Pág. 288

(32) Robin West es una excepción en este caso. Véase Robin L. West, The Authoritarian Impulse in Constitutional Law, 42 U. MIAMI L. REV. 531, 534-39 (2988); Robin L. West, Constitutional Scepticism, 72 B.U. L. REv. 765, 780-92 (1992); Robin West, Progressive and Conservative Constitutionalism, 8 MICH. L. Rev. 642, 672-77 (1990); Robin West, Reconstructing Liberty,59 TENN. L. REV. 44 I (2992); Robin West, Progressive constitutionalism: reconstructing the fourteenth amendment (1994). 
La fe depositada en el texto constitucional permite creer que las disposiciones escritas serán realidades futuras. Si partimos de la premisa de que las ideas tienen vida propia y una vez que un concepto se arraiga no existe una manera para prever a dónde nos conducirá o qué resultados se obtendrán a partir de su formulación (Hodes, 1970), podemos creer en que la preexistencia del texto constitucional establece el concepto, pero es la interpretación y los cambios políticos los que permiten que estos abarquen temas relacionados con las mujeres.

La fidelidad a la constitución se mantiene, no como una expectativa sin fundamento, sino como una creencia en el cambio que nace a partir de hacer una lectura crítica al texto constitucional ${ }^{(33)}$. No obstante, la interpretación no es el único camino para lograr garantías para las mujeres, su participación en la política o en las ramas del poder público permite que estas empleen un camino diferente para alcanzar la transformación.

Dentro de la corriente que piensa en la jurisprudencia como campo donde se desarrolla el feminismo constitucionalista, se presentan preguntas acerca de: ¿Cómo pueden los jueces conciliar la necesidad de tener en cuenta el contexto y las preocupaciones acerca de la consistencia y la predictibilidad? y ¿Cuál es el rol que cumple el contexto social en los juicios feministas en materia constitucional? Autores como Kenneth L. Karts (Karst, 1984: 480-507) inauguran una nueva forma de pensar la situación de las mujeres, no se preguntan sobre ¿qué puede hacer la constitución por las mujeres? Por el contrario, reinterpretan la pregunta y piensan: ¿qué es lo que las mujeres pueden hacer por la constitución? Este interrogante mantiene vigencia. Casi veinte años después de la publicación del trabajo de Karst, las feministas constitucionalistas aún se preguntan ¿cómo las constituciones moldean las vidas de las mujeres? y ¿cómo las mujeres moldean la constitución? Estas dudas encuentran respuesta al pensar en las mujeres como sujetos participantes en la jurisprudencia constitucional. En 2004 Beverley Baines y Ruth Rubio-Marin publicaron Gender and Jurisprudence, una colección de trabajos de autores pertenecientes a diferentes tradiciones jurídicas, en este libro exponen cómo las constituciones moldean la vida de las mujeres y como estas transforman las constituciones a través de la jurisprudencia. Es en estos trabajos donde se puede dilucidar el desempeño de las mujeres en la búsqueda de la inclusión, un ejemplo de ello es su participación ante los tribunales. En estos espacios las feministas encuentran desafíos complejos debido a que sus puntos de partida difieren -en ocasiones- de las posiciones judiciales, obligándolas a proponer mecanismos para persuadir a aquellos que tienen el poder de interpretar, esto señalando los vínculos entre lo que para ellos -los interpretes- es familiar y lo que es esencial para las mujeres. Es a través del litigio que las mujeres desarrollan y cambian el significado

(33) En este sentido ver: MacKinnon, C. A. (January 01, 1997). "Freedom from Unreal Loyalties": On Fidelity in Constitutional Interpretation. Fordham Law Review, 65, 4, 1773-1780. 
de las normas constitucionales. Igualmente, desde el estudio de la jurisprudencia constitucional, se ha tratado de evaluar el impacto de la actuación de las mujeres en casos específicos como participación política, derechos sexuales y reproductivos, matrimonio, discriminación, temas económicos, entre otros. Esta visión del feminismo como herramienta de análisis del derecho constitucional puede encontrarse en el artículo de Karst Kenneth Women's Constitution (1984), donde advierte que la idea de mujer es una construcción social reflejada y reforzada por el derecho, en su caso, el derecho norteamericano. Al pensar acerca del papel del derecho constitucional Kenneth propone una "Constitución de la Mujer" (Women's Constitution) no desde el diseño constitucional o cambios introducidos desde enmiendas o reformas, sino a través de la interpretación judicial de la constitución.

\section{Los tribunales como espacios de consecución de derechos, el caso de las feministas norteamericanas y los caminos a la igualdad}

La preocupación constitucional que por excelencia tienen las feministas norteamericanas versa sobre temas relacionados con la igualdad y la equidad, de manera específica la interpretación de la cláusula de igual protección en Estados Unidos. La igualdad ha sido uno de los temas centrales en el escenario jurídico norteamericano desde 1954 con la decisión Brown v. Board of Education, que dio inicio a una nueva etapa en el derecho constitucional estadounidense. Como consecuencia del protagonismo que adquirió la igualdad en este fallo y en decisiones subsecuentes, se presentó un cambio en el orden de los principios de aplicación predilectos de la Corte, el primero de ellos era la libertad. Al dar relevancia a la igualdad, en el caso Brown la Corte proporcionó un lugar privilegiado a este principio, incluso por encima de derechos como la libertad de asociación, mostrando con ello que la libertad no era el único valor fundamental de la Constitución. Durante años la Corte empleó la igualdad como herramienta para dar un nuevo aire a la interpretación judicial del texto constitucional. Los casos estadounidenses que versan sobre igualdad son ricos en intervenciones feministas, siendo posible identificar un método de participación e intervención en el espacio constitucional, el cual consistiría en la selección cuidadosa e interpretación de casos que permiten que la Constitución -sin ser modificada- sea interpretada a la luz del trato igualitario y digno que necesitan las mujeres.

En efecto, la Enmienda Decimocuarta a la Constitución de los Estados Unidos establece la cláusula de igual protección que prohíbe a los Estados negarle a cualquier persona que se encuentre dentro de sus límites jurisdiccionales la misma protección de las leyes. En otras palabras, las leyes de los estados deben ser aplicadas de manera homogénea a los individuos que se encuentren en condiciones y circunstancias similares. Una violación a esta disposición podría ocurrir, por ejemplo, en el caso en el que un estado prohíba por razones de raza 
que un individuo desempeñe un trabajo específico ${ }^{(34)}$. No obstante, esta enmienda no tiene la intención de propender por la "igualdad" entre individuos o clases, lo que busca es la "igual aplicación" de las leyes. Al negar a los Estados federados la posibilidad de discriminar, a ciertas personas, esta disposición resulta crucial para la protección de los derechos civiles. La enmienda consagra lo siguiente:

\section{Enmienda XIV \\ (Ratificada el 9 de julio de 1868)}

Sección 1. Todas las personas nacidas o naturalizadas en los Estados Unidos y sometidas a sujurisdicción son ciudadanos de los Estados Unidosy de los Estados en que residen. Ningún Estado podrá dictar ni dar efecto a cualquier ley que limite los privilegios o inmunidades de los ciudadanos de los Estados Unidos; tampoco podrá Estado alguno privar a cualquier persona de la vida, la libertad o la propiedad sin el debido proceso legal; ni negar a cualquier persona que se encuentre dentro de sus límites jurisdiccionales la misma protección de las leyes. ${ }^{(35)}$

Al respecto, la Corte Suprema de los Estados Unidos y la doctrina constitucional indican como reglas que se desprenden de la cláusula de igual protección (i) la exigencia de igualdad en el contenido de las disposiciones legislativas que emanan de autoridades públicas, y (ii) el principio de antidiscriminación (antidiscrimination principle) por parte de los poderes públicos (Martin, 2003).

La pregunta sobre ¿cuándo se presenta una violación a la cláusula de igual protección? surge en el momento en que un Estado otorga a ciertas personas el derecho a ejercer una actividad mientras le niega a otros individuos el mismo derecho. Sin embargo, la jurisprudencia no ha establecido una regla clara para decidir cuándo una determinada clasificación resulta inconstitucional. No obstante lo anterior, la Corte Suprema de los Estados Unidos ha implementado el uso de diferentes test, que dependen del tipo de clasificación que emplea la norma sometida a discusión y la vulneración a derechos fundamentales que sea alegada ${ }^{(36)}$.

(34) Cfr. Cornell University Law School. Equal Protection: An Overview. sitio Web de la Escuela de Derecho de la Universidad de Cornell: http://www.law.cornell.edu/wex/equal_protection. Sobre el significado de la equal protection clause. Michael Rosenfeld cita las palabras del juez Renhquist de la Corte Suprema de los Estados Unidos: "La cláusula de igualdad no significa que todas las personas deben ser tratadas del mismo modo. Mejor dicho, su principio general es que la persona situada como similar debe ser tratada de forma similar". M. Rosenfeld, Conceptos Clave y delimitación del ámbito de análisis de las acciones afirmativas, en Acciones Afirmativas, [en línea] disponible en: http://www.conapred.org.mx/ documentos_cedoc/AA_MSJ.pdf

(35) En su ensayo Grupos y la Cláusula de Igual Protección, Owen Fiss señala que esta cláusula fue diseñada para la protección de los grupos afrodescendientes. Sin embargo, indica que la vaguedad del termino personas dispuesto en la enmienda XIV tiene como efecto que otros grupos invoquen la protección constitucional. Fiss, O. (1999). Grupos y la Cláusula de la igual protección. En Gargarella, R. Derecho y grupos desaventajados (pp. 137 - 167). Barcelona: Gedisa.

(36) Tradicionalmente, la Corte Suprema encuentra constitucional la clasificación que realizan los estados si estas cuentan con una base racional (rational basis) a un propósito legítimo del estado. En otros 
Para que la clasificación realizada por un Estado sea admitida como constitucional bajo los parámetros del test, debe probarse que el Estado tiene un interés superior en la norma y que la clasificación es necesaria para alcanzar dicho interés. Se aplica el test de escrutinio estricto en las leyes que interfieren con derechos fundamentales como los consagrados en la primera enmienda o el derecho a la privacidad. Las clasificaciones que tienen como base el género exigen demostrar más que una base racional, aunque en estos casos no se haga uso de un escrutinio estricto. Los distintos tipos de escrutinio aparecieron por primera vez en la nota al pie $4^{(37)}$ del caso Estados Unidos v. Carolene Products ${ }^{(38)}$. Este caso permitió el desarrollo de las diferentes modalidades de test para evaluar la violación a la cláusula de igual protección ${ }^{(39)}$ :

No es necesario examinar ahora si la legislación que restringe los procesos políticos que normalmente debieran lograr la derogación de legislación indeseable debe ser sometida a un escrutinio judicial más exigente en virtud de las prohibiciones generales de la Decimocuarta Enmienda que otros tipos de legislación...

Tampoco es necesario que nos preguntemos si consideraciones similares deben ser incluidas en la revisión de leyes dirigidas a alguna minoría religiosas, nacionales, o racial en particular: es decir, si los prejuicios contra minorías marginales e insulares puede ser una condición especial, que tienda seriamente a limitar el funcionamiento de los procesos políticos que normalmente debe ser esperado que protejan a las minorías, y que puede requerir una disquisición judicial correspondientemente más inquisitiva. ${ }^{(\mathbf{4 0})}$ (Cursivas del texto citado).

\footnotetext{
casos, la Corte ha empleado un análisis más riguroso haciendo uso del escrutinio estricto (strictly scrutinize) cuando se está en presencia de una clasificación sospechosa (suspect classification). Para hacer uso de este criterio, se debe probar que la ley del estado o la administración tienen como propósito discriminar. Por ejemplo, si el test arroja como resultado que una norma hace distinciones de raza, nacionalidad, o leyes que discriminan a ciudadanos no norteamericanos, se hará uso de un escrutinio estricto por considerar que dichas regulaciones contienen un criterio sospechoso de clasificación.
}

(37) La más celebrada de las notas al pie del derecho constitucional. Ver: Powell, L. F. (October 01, 1982). "Carolene Products" Revisited. Columbia Law Review,82, 6, 1087-1092. p. 1087.

(38) United States v. Carolene Prods. Co., 304 U.S. 144, 152 n.4 (1938). En esta decisión de la Corte Suprema de los Estados Unidos conoce el caso de una ley federal que regulaba la leche con aditivos, en la misma se emplea un análisis con un nivel de escrutinio mínimo a la regulación económica. Sin embargo, el caso es relevante debido a que contiene la nota al pie 4 donde se exponen niveles diferentes de escrutinio judicial a otros tipos de casos. Powell señala que dicha nota al pies ha sido referenciada por la Corte Suprema en más de 28 ocasiones. "Carolene Products retains its fascination solely because of Footnote 4- the most celebrated footnote in constitutional law". Powell, L. F. (October 01, 1982). "Carolene Products" Revisited. Columbia Law Review,82, 6, 1087-1092. p. 1087.

(39)

(40) Traducción tomada de: Yoshino, K. El closet judicial y el altar legislativo. En: Seminario en Latinoamérica de Teoría Constitucional y Política, \& Alegre, M. (2010).Derecho y sexualidades: [SELA 2009]. Buenos Aires: Libraría. Artículo [en línea] disponible en: http://www.law.yale.edu/documents/pdf/Student_Organizations/SELA09_Yoshino_Sp_PV.pdf 
En la actualidad, la Corte Suprema no ha definido el contenido y alcance de la cláusula de igual protección. Sin embargo, los académicos han ofrecido diferentes modelos para su aplicación. La doctrina contrasta dos concepciones de igual protección, por un lado, se enfocan en el tratamiento igualitario ante la ley y por el otro, se observa si los resultados de la aplicación de la norma son iguales.

De acuerdo con el primer caso, es decir el de tratamiento igualitario, el gobierno trata a las personas de igual manera sin discriminación alguna, si en efecto se prueba que la ley no tiene como propósito fundante la discriminación, entonces no importa si el resultado es la desigualdad. Mientras tanto, el enfoque de la igualdad en el fin enfatiza en los resultados de las acciones del gobierno. Por ejemplo, la discusión acerca de si la prueba de los resultados discriminatorios de una ley es suficiente para establecer una violación al debido proceso sustancial, tendría dos formas de solucionarse. Aquellos que apoyan el enfoque del tratamiento igualitario podrían afirmar que no se está frente a una violación a la cláusula de igual protección ya que debe probarse el propósito de discriminación que tiene la ley, en contraste, el enfoque de la igualdad en resultados encontraría que es posible invocar la sanción, aunque la ley sea neutral debido al impacto discriminatorio que tendría la medida.

Para el análisis de esta cláusula se inicia con la siguiente pregunta básica: ¿Es la clasificación realizada por el gobierno justificada por un propósito suficiente? Por otro lado, los asuntos que versan sobre igual protección pueden descomponerse en las siguientes cuestiones, a saber: (a) ¿cuál es la clasificación que se emplea? En este caso existen dos maneras para probar la clasificación, primero, demostrando que existen de cara al derecho o probando que si bien la ley es ostensivamente neutral, la aplicación de la misma tiene como consecuencia un impacto discriminatorio y un propósito discriminatorio;(b) ¿Cuál es el nivel de escrutinio que debe emplearse? Los diferentes tipos de escrutinio serán aplicados dependiendo del tipo de discriminación: escrutinio estricto, intermedio y de base racional.

En el escrutinio estricto la ley se mantiene siempre que la discriminación sea necesaria para alcanzar un propósito convincente por parte del gobierno, el escrutinio intermedio se acepta cuando el gobierno demuestra un objetivo importante en la ley no teniendo que ser apremiante. El test de base racional requiere que el gobierno demuestre que está legitimado para hacer dicha clasificación y finalmente, la duda acerca de si ¿la acción tomada por el gobierno cumple o no con el nivel de escrutinio. Para que cumpla con el escrutinio estricto el fin de la ley debe ser convincente, para el intermedio el fin debe ser importante y para la prueba de base racional debe ser un propósito legítimo ${ }^{(41)}$.

(41) La discusión en este punto se centra en los debates que surgen desde aquellos que afirman que la Corte no tiene tres niveles de escrutinio sino que tiene una gama de normas, y los que demandan una escala móvil de revisión (sliding scale of review). No obstante las criticas anteriores, este test ha sido mantenido por la Corte a lo largo de los años, sin embargo algunos teóricos hablan de la existencia de un nuevo estándar constitucional el excedengly persuasive justification de equal protection, este test 
Reva B. Siegel en sus artículos El discurso de la igualdad: Los valores de la antisubordinación y la anticlasificación en las luchas constitucionales sobre el caso Brown (Siegel, 2004: 1470-1547) y Why Equal Protection No Longer Protects: The Evolving Forms Of Status- Enforcing State Action (Siegel, 1997: 1111-1148) explora la relación que existe entre los principios y la política constitucional, a traves de la forma en la que se habla del significado de la decisión judicial. Para esta autora, es posible identificar en la Enmienda XIV el principio anticlasificación, calificando este como un artefacto de las luchas políticas sobre la implementación de la decision Brown. Afirma además, que este principio varía en el tiempo debido a que es aplicable para reivindicar diferentes tipos de asuntos sociales, entendiendo asi por qué Brown no solo funciona para el movimiento de los derechos civiles, sino que además le sirve al movimiento de mujeres y sus incursiones en temas de igualdad. En este mismo sentido, la jurisprudencia de la antisubordinacion, de acuerdo con Siegel:

"... se ve reflejada en la historia de los debates acerca de Brown donde muestra cómo los conflictos raciales acechan los silencios, ambigüedades y conflictos de la moderna doctrina de igual protección. Finalmente, es una historia que sugiere el modo en que los contornos de los principios constitucionales surgen del crisol de la política constitucional."(42)

Siegel muestra cómo los valores de la antisubordinación están contenidos en el origen del principio de anticlasificación, sin embargo, este principio debe ser ponderado ante la necesidad de contar con una constitución que sirva para gobernar a todos. Dos casos de la Corte Suprema: Brown v. Board of Education y Lawrence v. Texas son entendidos por Siegel como “... una intervención trascendental respecto de un conjunto de arreglos sociales controvertidos, cuya lógica ultima y aplicación práctica todavía permanecen confusas." Para este discurso sobre la antisubordinación y la antidiscriminación, la autora se apoya en Owen Fiss al entender que la antidiscriminacion no es suficiente para entender la enmienda XIV, lo cual conduce a un avance en la interpretacion que va más alla de dicho principio. Pero más allá de un debate sobre la interpretación, los casos no son elementos estaticos del derechos constitucional. Estos fallos sirven como piedra angular para la moviliazión de las mujeres para que sus voces no sean olvidadas. ${ }^{(43)}$ Los efectos

\footnotetext{
sería más riguroso que el intermediate test pero no alcanzaría los niveles del stric scrutiny test. En este sentido ver: Fernando Rey Martínez. Jurisprudencia norteamericana reciente sobre acciones afirmativas basadas el género. Aequalitas: Revista Jurídica de Igualdad de Oportunidades entre Mujeres y Hombres, num. 4, 2000, págs. 8 y sigs. En sentido contrario, ver: Peter Lurie: "The law as they found it: disentagling gender-based affirmative action programs from Croson", The University of Chicago Law Review, vol. 59. 1992- II, págs. 1587-1588. Citado en: Martin Vida, María Ángeles. Evolución del principio de igualdad en Estados Unidos. Nacimiento y desarrollo de las medidas de acción afirmativa en derecho estadounidense (2003). Revista Española de Derecho Constitucional. Año 23. Núm. 68. Mayo-Agosto.

(42) Siegel, R. B. (March 01, 2004). Equality Talk, op. cit., p. 1547.

(43) Ver:Williams, Wendy., The Equality Crisis: Some Reflections on Culture, Courts, and Feminism, 7 Women's Rts. L. Rptr. 175 (1982).
} 
de los fallos, como lo señala Siegel permiten -por ejemplo- la interpretación moderna de la Constitución de los Estados Unidos en temas que afectan los arreglos de género tradicionales en la familia (Siegel, 2005: 306-332).

\section{Conclusión}

El feminismo constitucional se manifiesta de numerosas maneras, a través del estudio de casos específicos como los derechos sexuales y reproductivos, la participación de las mujeres en las discusiones constitucionales, derechos laborales, entre otros. Si bien, en ocasiones no se presenta de manera expresa, existen esfuerzos desde diferentes campos del saber para promover cambios en las vidas de las mujeres, esos esfuerzos frecuentemente emplean las herramientas del derecho constitucional. De igual forma, el diseño constitucional y la interpretación judicial son estrategias del feminismo para promover una agenda constitucional inclusiva, pues permiten la coexistencia de diferentes proyectos de vida, esto dentro de un marco de igualdad y protección de las mujeres. Tanto el diseño como la interpretación constitucional son medios que le permiten a las mujeres su participación en esferas que habían permanecido ajenas a ellas durante mucho tiempo. En un espacio donde el derecho ha ayudado a que las mujeres sean oprimidas, es también el derecho el camino para cambiar el status quo.

A través del diseño constitucional, las mujeres participan en la creación de las normas que regularán su actuar y los derechos que las protegerán, sin embargo, la preocupación radica en el cumplimiento de la norma. ¿Cuáles son los medios para garantizar que las mujeres recibirán lo prometido? En la actualidad, los procesos constitucionales se caracterizan por ser un espacio de discusión abierto donde se busca la participación democrática de todas las fracciones de la sociedad. Las feministas aprovechan estos escenarios ya que entienden las constituciones y el cambio constitucional como el centro de la estructura institucional y el sistema legal de los estados. El cambio constitucional es visto como una estrategia para ampliar los derechos de género, la participación de las mujeres en espacios democráticos, abiertos y transparentes, contribuyen a que el resultado de la discusión constitucional aumente al incremento en las posibilidades de inclusión para mujeres.

Si bien las presento como dos categorías individuales, el diseño y la interpretación constitucional se encuentran unidas, ya que a través de la interpretación es posible complementar, ejecutar y desarrollar los objetivos planteados desde el diseño. Las múltiples estrategias que desarrollan las mujeres para ser incluidas en los espacios tradicionales de exclusión permiten que el camino hacia la igualdad de hombres y mujeres esté cada día más cerca. Pero no es solo en el mundo jurídico donde se deben dar las discusiones sobre el rol de la mujer, otros ámbitos deben ser explorados. 


\section{Bibliografía}

ADAMS, J., Adams, A., \& Adams, C. F. (1876). Familiar letters of John Adams and his wife Abigail Adams, during the Revolution: With a memoir of Mrs. Adams. New York: Hurd and Houghton.

AGOSÍN, M. (2001). Women, gender, and human rights: A global perspective. New Brunswick, NJ: Rutgers University Press.

AMAR, A. R. (1995). "Women and the Constitution" 18 Harvard Journal of Law and Public Policy 465.

BAINES, Beverley y Rubio-Marin, Ruth (eds) (2004), The Gender of Constitutional Jurisprudence, Cambridge University Press.

BAINES, Beverley, Barak-Erez, Daphne y Kahana, Tsvi (eds) (2012), Feminist Constitutionalism, Cambridge University Press.

BEVERLEY Baines, Ruth Rubio-Marin, Peter Oliver, Patrick Macklem, \& Nathalie Des Rosiers. (2017). Part VI Constitutional Theory, C Key Debates in Constitutional Theory, Ch.45 Feminist Constitutionalism in Canada. In The Oxford Handbook of the Canadian Constitution (Oxford Handbooks, p. Oxford Handbooks, The Oxford Handbook of the Canadian Constitution). Oxford University Press.

BARAK-EREZ, D. (2012). Her-meneutics. Feminist constitutionalism: Global perspectives, 85-97.

CACIOPPO, M. (1992-1993) Women and the Constitution; 19 Ohio N.U. L. Rev. 691.

DOBROWOLSKY, Alexandra y Hart, Vivien (eds) (2003), Women Making Constitutions: New Politics and Comparative Perspectives, Palgrave Macmillan.

---. (2003). Women, Constitutionalism and Contestation: Some Tentative Conclusions. Women making constitutions: New politics and comparativeperspectives. Houndsmill, Basingstoke, Hampshire: Palgrave Macmillan.

FALK, R. A., Johansen, R. C., \& Kim, S. S. (1993). The Constitutional foundations of world peace. Albany, N.Y: State University of New York Press.

FISS, Owen M. (1999). Grupos y la Cláusula de la igual protección. En Gargarella, R. Derecho y grupos desaventajados (pp. 137 - 167). Barcelona: Gedisa.

N. GARAY (2013). Las Declaraciones de los Derechos de la Mujer y la Ciudadana de 1791 y de Independencia de Haití de 1804 en el proceso de positivización de los derechos fundamentales. Aportes desde el constitucionalismo feminista, en Revista Letras Jurídicas, 28, México: Universidad Veracruzana. 
HART, Vivien, Democratic Constitution Making 10 (U.S. Inst. Of Peace, Special Rep. 107, July 2003), disponible en: http:// www.usip.org/pubs/specialreports/sr107/html

HIGGINS, T. E. (January 01, 1997). Democracy and Feminism. Harvard Law Review, 110, 8, 1657.

HODES, W. W., Women and the Constitution: Some Legal History and a New Approach to the Nineteenth Amendment, 25 Rutgers L. Rev. 26 (1970).

INTERNATIONAL IDEA, Marginalized Groups and Constitution Building (Stockholm: International IDEA, 2014).

IRVING, Helen (2008), Gender and the Constitution: Equity and Agency in Comparative Constitutional Design, Cambridge University Press.

---. (2011). Drafting, Design and Gender. Comparative Constitutional Law, 19-37.

ISHWARA BHATT, P. Constitutional Feminism: An Overview (2001) 2 SCC Journal (J).

JACKSON, Vicki (2009), 'Conclusion: Gender Equality and the Idea of a Constitution: Entrenchment, Jurisdiction, Interpretation', en Susan H. Williams (ed.), Constituting Equality: Gender Equality and Comparative Constitutional Law, Cambridge University Press.

JARAMILLO SIERRA, I.C. (2000). 'La crítica feminista al derecho', en Género y teoría del derecho, Siglo del Hombre Editores, Instituto Pensar y Facultad de Derecho de la Universidad de los Andes, Bogotá.

---. (2007). El reto de pensar la distribución: a propósito del libro de Janet Halley Split decisions: how and why to take a break from feminism. En: Colombia Revista de Estudios Sociales, Universidad de Los Andes, v.28 fasc.1 p.132 - 137.

KARST, Kenneth L. (1984). Woman's Constitution. (Duke Law Journal.) Duke University School of Law.

LUCAS, L. E. (January 01, 2009). Does Gender Specificity in Constitutions Matter (Note). Duke Journal of Comparative \& International Law, 20, 1, 133-166.

LUTZ, D. S. (2006). Principles of constitutional design. Cambridge: Cambridge University Press.

LURIE, Peter, The law as they found it: disentagling gender-based affirmative action programs from Croson , The University of Chicago Law Review, vol. 59. 1992- II, págs. 1587-1588.

MACKINNON, C. A. (January 01, 1997). “Freedom from Unreal Loyalties”: On Fidelity in Constitutional Interpretation. Fordham Law Review, 65, 4, 1773-1780. 
MACKINNON, C. A. "Gender in Constitutions." In The Oxford Handbook of Comparative Constitutional Law, edited by M. Rosenfeld and A. Sajó, 397-416. Oxford: Oxford University Press, 2012.

MARTIN VIDA, María Ángeles, Evolución del principio de igualdad en Estados Unidos. Nacimiento y desarrollo de las medidas de acción afirmativa en derecho estadounidense (2003). Revista Española de Derecho Constitucional. Año 23. Núm. 68. Mayo-Agosto.

MONOPOLI, Paula (2006), 'Gender and Constitutional Design', The Yale Law Journal 115: 2643.

MORGAN, Martha I. \& Monica Maria Alzate Buitrago, Constitution-Making in a Time of Cholera: Women and the 1991 Colombian Constitution, 4 Yale J. L. \& Feminism 353, 370-71 (1992).

POWELL, L. F. (October 01, 1982). "Carolene Products" Revisited. Columbia Law Review,82, 6, 1087-1092. p. 1087.

REY MARTÍNEZ, Fernando, Jurisprudencia norteamericana reciente sobre acciones afirmativas basadas el género. Aequalitas: Revista Jurídica de Igualdad de Oportunidades entre Mujeres y Hombres, num. 4, 2000, pág. 8 y sigs.

ROSENFELD, M., \& Sajó, A. (2012). The Oxford handbook of comparative constitutional law. Oxford, U.K: Oxford University Press.

ROSENFELD, M, en András, S., \& Catharine, A. M. (May 17, 2012). Gender in Constitutions.

RICCI, S, "Rethinking Women and the Constitution: An Historical Argument for Recognizing Constitutional Flexibility with Regards to Women in the New Republic" (2009) William \& Mary Journal of Women and the Law, Vol. 16. Artículo 8, 205-235.

SIEGEL, R. (May 01, 1997). Why Equal Protection No Longer Protects: The Evolving Forms of Status-Enforcing State Action. Stanford Law Review, 49, 5, 1111-1148.

---. (March 01, 2004). Equality Talk: Antisubordination and Anticlassification Values in Constitutional Struggles over Brown. Harvard Law Review, 117, 5, 1470-1547.

---. (January 01, 2005). Gender and the United States Constitution: Equal Protection, Privacy, and Federalism. Gender of Constitutional Jurisprudence, 306-332.

SKEET, Charlotte (2007), 'Gender and "Modern" Constitutionalism: The Treaty Establishing a Constitution for Europe', Northern Ireland Legal Quarterly 58: 142.

SULLIVAN, K (2002), 'Constitutionalizing Women's Equality', California Law Review 90: 735. 
SUNSTEIN, Cass R. (2001), Designing Democracy: What Constitutions Do, Oxford University Press.

WAYLEN, G. (2013). The Oxford handbook of gender and politics. Oxford: Oxford University Press. Pág. 589.

WEST, R. L. (February 01, 1990). Progressive and Conservative Constitutionalism. Michigan Law Review, 88, 4, 641-721.

---. (1992). Constitutional Scepticism, 72 B.U. L. Rev. 765, 780-92.

WAYLEN, G. (January 01, 2006). Constitutional engineering: What opportunities for the enhancement of gender rights? Third World Quarterly, 277, 1209-1221.

WHITTINGTON, K. E., Kelemen, R. D., y Caldeira, G. A. (2008). The Oxford handbook of law and politics. Oxford: Oxford University Press.

WILLIAMS, W, The Equality Crisis: Some Reflections on Culture, Courts, and Feminism, (1982) 7 Women's Rts. L. Rptr. 175.

YOSHINO, K. El closet judicial y el altar legislativo. En: Seminario en Latinoamérica de Teoría Constitucional y Política, \& Alegre, M. (2010).Derecho y sexualidades: [SELA 2009]. Buenos Aires: Libraría. 